\title{
Monitoring activity of hip injury patients (MoHIP): a sub-study of the World Hip Trauma Evaluation observational cohort study
}

Laura C. Armitage ${ }^{1 *}$ (D), Yuan Chi ${ }^{2}$, Mauro Santos ${ }^{2}$, Beth K. Lawson ${ }^{1}$, Carlos Areia ${ }^{3}$, Carmelo Velardo², Peter J. Watkinson ${ }^{3}$, Lionel Tarassenko ${ }^{2}$, Matthew L. Costa ${ }^{4}$ and Andrew J. Farmer ${ }^{1}$

\begin{abstract}
Background: Hip fracture is common, affecting $20 \%$ of women and $10 \%$ of men during their lifetime. The trajectory of patients' recovery as they transition from the acute hospital setting to their usual residence is poorly understood. Recently, the use of activity trackers to monitor physical activity during recovery has been investigated as a way to explore this trajectory.

Methods: This prospective observational cohort study followed patients from hospital to home as they recovered from a hip fracture. Participants were recruited from a single centre and provided with a 3-axis logging accelerometer worn as a pendant, for 16 weeks from recruitment. Participants received monthly follow-up visits which included questions about wearing the monitor. Monthly activity monitor data were also downloaded. Participant activity was estimated from the monitor data using the calibrated "Euclidean Norm Minus One" (ENMO) metric. Polynomial mixed-effects modelling was used to evaluate the difference between the weekly activity trends of 2 groups of participants: those with and without independent mobility at 16 weeks (defined by whether aids or personal assistance were required to mobilise).

Results: Twenty-nine participants from 125 eligible patients were recruited. Of these, 19 (66\%) reported being aware of wearing the monitor at least some of the time. Fourteen (48\%) participants withdrew before study completion. Data for thirteen (45\%) participants were of sufficient quantity to be included in the activity modelling procedure. Of these, 8 reported independent mobility at 16 weeks post-surgery, and 5 did not. By week 7 , the weekly predicted mean ENMO (ENMOW) values were significantly different between the two participant groups, demonstrating feasibility of the model's ability to predict which patients will report independent mobility at 16 weeks.

\footnotetext{
* Correspondence: laura.armitage@phc.ox.ac.uk

${ }^{1}$ Nuffield Department of Primary Care Health Sciences, Radcliffe Primary Care Building, Radcliffe Observatory Quarter, University of Oxford, Woodstock Road, Oxford OX2 6GG, UK

Full list of author information is available at the end of the article
}

(c) The Author(s). 2020 Open Access This article is licensed under a Creative Commons Attribution 4.0 International License, which permits use, sharing, adaptation, distribution and reproduction in any medium or format, as long as you give appropriate credit to the original author(s) and the source, provide a link to the Creative Commons licence, and indicate if changes were made. The images or other third party material in this article are included in the article's Creative Commons licence, unless indicated otherwise in a credit line to the material. If material is not included in the article's Creative Commons licence and your intended use is not permitted by statutory regulation or exceeds the permitted use, you will need to obtain permission directly from the copyright holder. To view a copy of this licence, visit http://creativecommons.org/licenses/by/4.0/. The Creative Commons Public Domain Dedication waiver (http://creativecommons.org/publicdomain/zero/1.0/) applies to the data made available in this article, unless otherwise stated in a credit line to the data. 
(Continued from previous page)

Conclusions: This is the first study to our knowledge to investigate acceptability and feasibility of a pendant-worn activity monitor in this patient cohort. Acceptability of wearing the monitor and feasibility of recruitment and retention of participants were limited. Future research into the use of activity monitors in this population should use minimally intrusive devices which are acceptable to this population.

Study registration: MoHIP is a sub-study of the World Hip Trauma Evaluation (WHiTE) Study (ISRCTN 63982700).

Keywords: Hip fracture, Rehabilitation, Activity, Monitoring

\section{Background}

Hip fracture is a common injury, affecting $20 \%$ of women and $10 \%$ of men during their lifetime. In 2018, the James Lind Alliance identified the top two research priorities in fragility fracture of the lower limb as firstly understanding the best in-hospital and secondly best out-of-hospital physiotherapy regimes for recovery [1]. However, little is known about the trajectory of recovery in mobility as patients transition from hospital to home. More recently, the use of activity trackers to monitor motion and physical activity during recovery from hip fracture is being investigated as a way to explore this trajectory $[2,3]$.

In 2018, the UK National Hip Fracture Database Report revealed that just $38.4 \%$ patients receive follow-up of their mobility at 120 days post injury [4] and the UK 'Hip Sprint' audit revealed that only $20 \%$ of hospitals achieved continuity of rehabilitation as patients transitioned to home [5]. Most people who sustain a hip fracture will have completed their rehabilitation by 4 months [5]. Research investigating the recovery of mobility during hip fracture rehabilitation has traditionally relied upon patient selfreport questionnaires, collected intermittently and at long intervals and as such are vulnerable to biases of selfreport, recall and social desirability [6].

There is a need for more detailed and objective information concerning recovery of patient mobility following a hip fracture, particularly at the critical point of transition from hospital to home, where continuity of rehabilitative care is frequently not achieved. Small and lightweight activity monitors provide an opportunity to measure the recovery of mobility with greater objectivity and frequency in this population.

The overall aim of this study was to assess feasibility and acceptability of collecting individual-level activity data via a wearable activity monitor worn on a pendant, to better understand the recovery of mobility in patients during the first 4 months following hip fracture. Study objectives were as follows:

1) Investigate the acceptability of wearing the activity monitor for the duration of the 4-month follow-up period through participant self-report about wearing the monitor
2) Assess feasibility of using 3-axis logging accelerometers to collect activity data in this population by:

a) Assessment of participant recruitment

b) Assessment of participant retention rate

c) Investigating whether an algorithm can be developed and applied to analyse low-intensity raw activity data obtained from the monitors.

d) Investigating whether this can appropriately differentiate between those participants who report independent mobility versus requiring assistance with mobility at 16 weeks.

\section{Methods \\ Study design}

This prospective observational cohort study followed participants from hospital to home for 16 weeks as they recovered from a hip fracture injury. Participants were recruited from a single centre (John Radcliffe Hospital, Oxford, UK) from May 2018 to August 2019. At the point of recruitment, participants were provided with a 3-axis logging accelerometer, the AX-3 (Axivity Ltd, Newcastle upon Tyne, UK), worn within a pouch attached to a pendant as shown in Fig. 1. A pendant was chosen for two main reasons; firstly, with the monitor being capable of 3-axis data capture, we sought to investigate whether an algorithm could be developed to analyse low-intensity data with the monitor worn in the axial position, as previous studies have been limited to investigating this on an arm or leg $[2,3,7]$. Secondly, we aimed to investigate whether this would be an acceptable method of wear in an older population who are frequently familiar with personalised alarm systems [8] commonly worn as a pendant. Participants received a follow-up visit at their place of residence every 4 weeks for up to 16 weeks from recruitment. At each visit, a custom-made case report form was completed with the participant which included the following:

1. A 5-item mobility scale (Additional file 1)

2. A 3-item scale about awareness of wearing the activity monitor (Additional file 1)

3. A 5-item scale about how the participant felt about wearing the monitor (Additional file 1) 


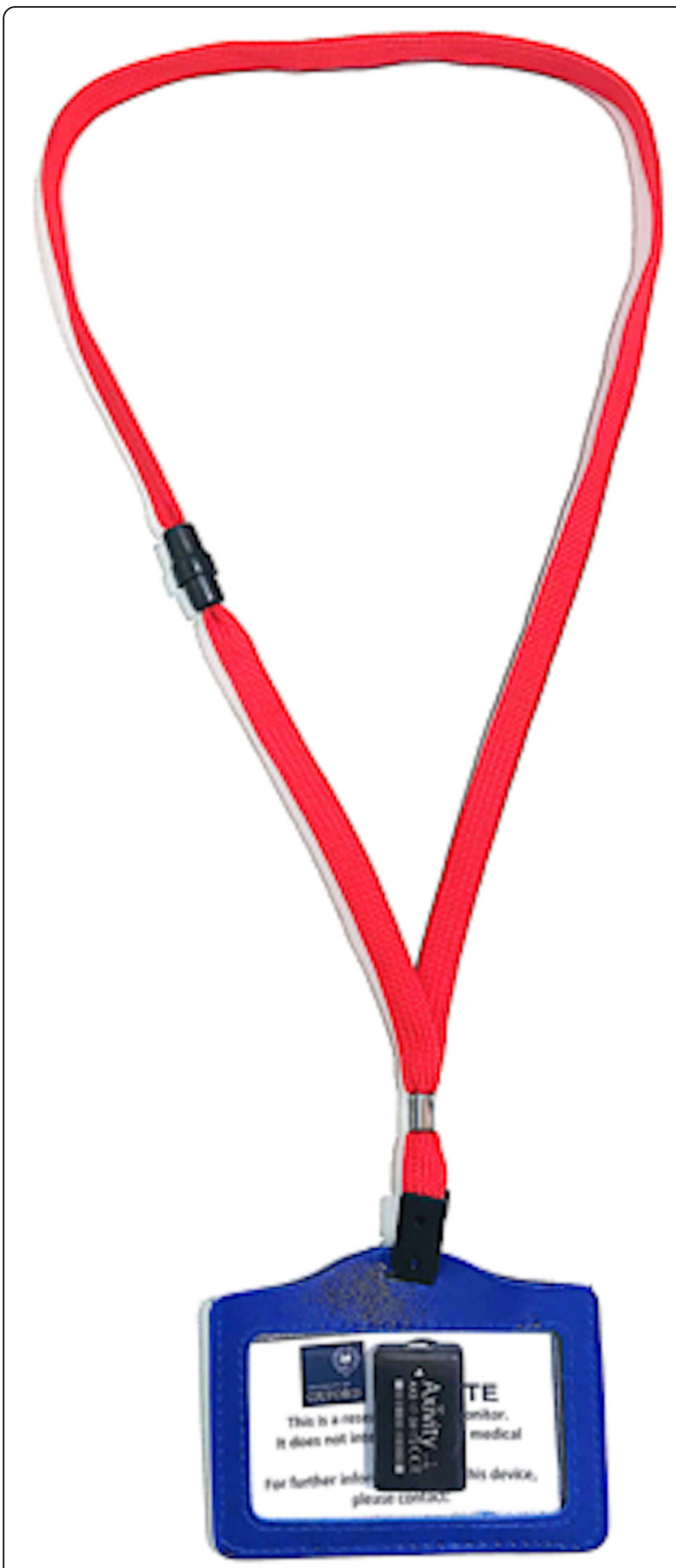

Fig. 1 Axivity Ltd AX3 monitor fitted in a pendant

At all visits (except final), the participant was supplied with a replacement, fully-charged, monitor.

\section{Participants}

\section{Eligibility}

Patients eligible for recruitment to MoHIP were those who met eligibility criteria for, and were recruited to, the
World Hip Trauma Evaluation (WHiTE) cohort study [9]. WHiTE is an observational study in which patients receive usual medical and rehabilitative care and are also followed up to collect the UK Core Outcome Set for research into hip fracture [10]. Inclusion criteria were the same as for the WHiTE study (age $\geq 60$ and receiving operative treatment for hip fracture) [9], and exclusion criteria specific to the MoHIP sub-study were as follows:

(i) Cognitive impairment

(ii) Discharged from hospital to a nursing home as permanent place of residence

(iii) Discharged to an area outside the hospital's neighbouring counties of Berkshire, Buckinghamshire and Oxfordshire.

Patients with cognitive impairment were not eligible for inclusion owing to the potential safety implications that might arise from prolonged wear of a pendant system to house the activity monitor. Participants who were discharged to a temporary rehabilitation facility were eligible for inclusion and received the same research follow-up visits as those who were discharged to their own homes.

\section{Consent}

Eligible patients were approached at the first appropriate time during the post-operative period when they had regained capacity (typically the first day postoperatively). They were provided with study information including detailed information about the monitor, its capability, and how it would be worn. Participants were provided with the opportunity to ask questions and discuss the study with the researcher, their family, and carers for as long as required. All patients were required to give written consent to both the WHiTE study and MoHIP sub-study to participate.

\section{Data sources}

Baseline data were collected for recruited participants including demographic data (age, sex), baseline comorbidities, pre-fracture mobility, type of fracture, type of anaesthesia, type of surgery and health status using the EuroQol 5-Dimension 5-Level measure (EQ-5D-5 L) [11].

\section{Activity monitoring}

Monitors were configured to record activity for 28 days at a sampling frequency of $25 \mathrm{~Hz}$ and range of $\pm 8 \mathrm{~g}$. This configuration enabled the capture of the movements of interest in this study, most in the range up to $12 \mathrm{~Hz}$ [12]. The activity monitor was replaced at each 4weekly follow-up visit by the primary care research nurse, and the monitor worn during the preceding 
month was returned to the study team for data download.

\section{Outcome measures}

The primary outcome measure was acceptability of using a CE-marked activity-monitoring device worn as a pendant, to assess participants' physical activity during their recovery from a hip fracture. Acceptability was measured according to:

i. Participant wear time

ii. Percentage participants who reported feeling positive about wearing the monitor half or more of the time.

We assessed feasibility of using the activity monitor devices to estimate activity in this patient population through:

i. Participant recruitment rates

ii. Completion of study protocol

iii. Identifying patterns of activity extracted from monitor data, looking for correlations with standard core outcome measures and investigating whether the activity data can identify patients who are inactive. Participants were required to have activity data for $\geq 50 \%$ ( $\geq 8$ weeks) of the study period to be included in this analysis.

A threshold of $80 \%$ is frequently considered an appropriate cut-off for assessing measures of acceptability and feasibility in pilot studies and was therefore adopted for this study [13]. For example, the wearing of the monitor could be considered acceptable if (i) $\geq 80 \%$ participants wore the monitor beyond 13 weeks of the 16-week study period ( $80 \%$ of the follow-up period) and (ii) $\geq 80 \%$ of participants reported feeling positive about wearing the monitor half or more of the time. Similarly, recruitment and retention of participants to this study could be considered feasible if (i) $\geq 80 \%$ eligible and approached participants were recruited and (ii) $\geq 80 \%$ of those recruited complete the study protocol.

\section{Sampling and risk of bias}

In order to limit sampling bias, all participants recruited to the WHiTE cohort study were screened for eligibility for MoHIP. Participants of all levels of baseline mobility and functional ability were screened for inclusion. Recruited participants were not provided with feedback regarding their activity levels, minimising risk of observation bias.

All participants recruited to the study received usual medical and rehabilitative care.

\section{Data management and statistical methods}

This study was designed to investigate acceptability and feasibility of the use of a wearable activity monitor in an older and complex patient population. We have estimated that with a sample size of 29 participants, we would be able to detect a $20 \%$ withdrawal rate with a $95 \%$ confidence interval of $\pm 12 \%$.

Study data were managed using REDCap electronic data capture tools hosted at the University of Oxford, providing a secure electronic data capture system $[14,15]$.

Figure 2 shows the block diagram of the accelerometer data pre-processing steps. Each participant's data was first filtered using an eighth-order Butterworth bandpass filter, with cut-off frequencies of $0.2 \mathrm{~Hz}$ (highpass) and $12 \mathrm{~Hz}$ (lowpass) [7]. A non-wear time metric was then applied to remove periods in which the tracker was not being used. This metric was defined as all the nonoverlapping 30-min windows in which the standard deviation (SD) was below $13 \mathrm{mg}$, in each of the three axes, or with an absolute value of less than $50 \mathrm{mg}$ in at least 2 of the axes. Additionally, given that most participants did not wear the activity monitors during the night, only the accelerometer data recorded between 06:30 and 21:30 were considered.

The "Euclidean Norm Minus One" metric, ENMO $=\max \left(0, \sqrt{x^{2}+y^{2}+z^{2}}-1\right)$, was used as a proxy for the patient activity $[7,16-18]$, in which $x, y$, and $z$ represent each of the axes of the accelerometer sensor; the standard gravity created at the Earth's surface $(1 g)$ is subtracted; and negative ENMO values are set to zero. The latter may be a result from calibration errors, noise or negligible body movement [16]. The ENMO (computed at the sensor's $25 \mathrm{~Hz}$ sampling rate) was averaged per minute, and then per week (from 1 to 16 postoperative weeks), for each participant. Each participant's average ENMO per week is denoted as ENMO thereafter.

\section{Mixed-effects model}

A polynomial mixed-effects model was used to analyse the association between the participants' ENMO $_{W}$ (dependent variable) and the number of post-operative recovery weeks and the mobility outcome, i.e. those with and without independent mobility (defined by whether aids or personal assistance were required to mobilise at 16 weeks). The latter was modelled as a fixed-effect (binary variable denoted "mobility" coded 1 for participants 


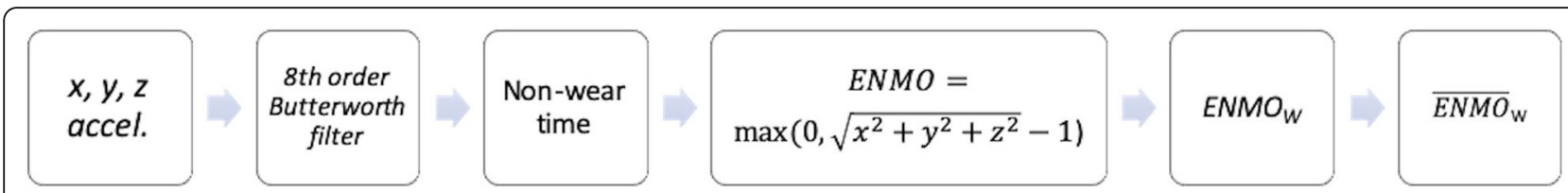

Fig. 2 Block diagram of the pre-processing steps for each participant's accelerometer data. ENMO is the calibrated "Euclidean Norm Minus One" metric. $\mathrm{ENMO}_{W}$ corresponds to each participant's average EMNO per week. ENMOOW are predictions resulting from a mixed-effects model derived from the participants' $\mathrm{ENMO}_{W}$ data

reporting independent mobility and 0 otherwise). Random-effects were considered at the participant level for the number of post-operative weeks variable (coded as "week"). A normal prior was considered for both the random-effects terms and for the model error term, and the model fit achieved by maximum likelihood estimation. The model polynomial order was varied from 1 to 5 , and the cubic model was found to have both the lowest Akaike and Bayesian Information Criteria. The latter is named "baseline model" $\left(\mathrm{ENMO}_{W}\right)$ thereafter, with the formula (in Wilkinson notation):

$$
\begin{aligned}
\mathrm{ENMO}_{W}= & 1+\text { mobility }+ \text { week }+ \text { week }^{2}+\text { week }^{3} \\
& +\left(\text { week }+ \text { week }^{2}+\text { week }^{3} \mid \text { patient }\right) .
\end{aligned}
$$

\section{Statistical analysis}

The Likelihood Ratio Test (LRT) was used to assess if the baseline model with an additional clinically relevant parameter (added as an interaction or an additional fixedeffects term) achieved a significantly better model fit. We identified three additional parameters for investigation. Firstly, receipt of physiotherapy in the first month post hospital discharge (coded as 1 for or out-of-hospital physiotherapy and 0 otherwise) which was selected due to the nationally low reported rates of continuity of physiotherapy upon discharge [5] and previously reported negative association of lack of post discharge physiotherapy on functional recovery [19]. Secondly we investigated the effect of self-report of anxiety or depressive symptoms at baseline assessment on model fit as this has previously been shown to be negatively associated with regain of physical independence following hip fracture [20]. Finally, we investigated the effect of baseline self-report of pain and discomfort during the hospital stay on the model as this has been previously shown to be negatively associated with both ability to mobilise in the early post-operative period [21] and functional regain of outdoor mobility post hospital discharge [22].

\section{Results}

Participants

Enrolment

Between May 2018 and August 2019, 125 of 382 participants recruited to the WHiTE Cohort Study were eligible for recruitment to MoHIP. The most common reason for exclusion was cognitive impairment $(n=171$, $44.7 \%)$. Of the eligible 125 participants, 81 (65\%) were approached and 29 (36\%) of these were recruited to MoHIP, meaning that the study did not meet the recruitment feasibility threshold of enrolling $\geq 80 \%$ of those eligible and approached. The flow diagram of screening and enrolment is shown in Fig. 3. Participant characteristics at baseline of the whole cohort and the thirteen participants included in the activity data modelling are presented in Table 1.

\section{Feasibility and acceptability of using the pendant-worn activity monitor for data collection Participant retention and experience of wearing the monitor}

Of the 29 recruited participants, 15 (52\%) wore the activity monitor for 13 weeks or more, but two of these participants had data missing for $\geq 50 \%$ ( 8 weeks) of the study period and were therefore ineligible for inclusion in the activity data modelling procedure. The study therefore did not achieve the feasibility thresholds of $\geq$ $80 \%$ participants wearing the monitor for $\geq 80 \%$ of the follow-up period. Of the 29 participants, 19 (68\%) reported being aware of the monitor at least some of the time. Fourteen (48\%) participants withdrew before study completion. Reasons for withdrawal broadly related to two themes: firstly, participants reported the pendant system "got in the way" or was too visible; secondly, some participants reported that ongoing study participation involving daily monitor use and monthly visits was too high a demand in the context of an already high medical burden.

Of the 15 participants who completed the study protocol, 2 (13\%) reported being unaware of the monitor during periods of wear, and 9 reported being aware of the monitor at least some of the time; 8 of these participants reported a negative awareness such as the monitor getting in the way, being too visible or irritating them. However, 7 (50\%) of the 14 participants who completed the study protocol reported feeling positive or very positive about wearing the monitor at half or more of their follow-up visits, and 3 participants reported feeling positive or very positive about wearing the monitor throughout the study period. 


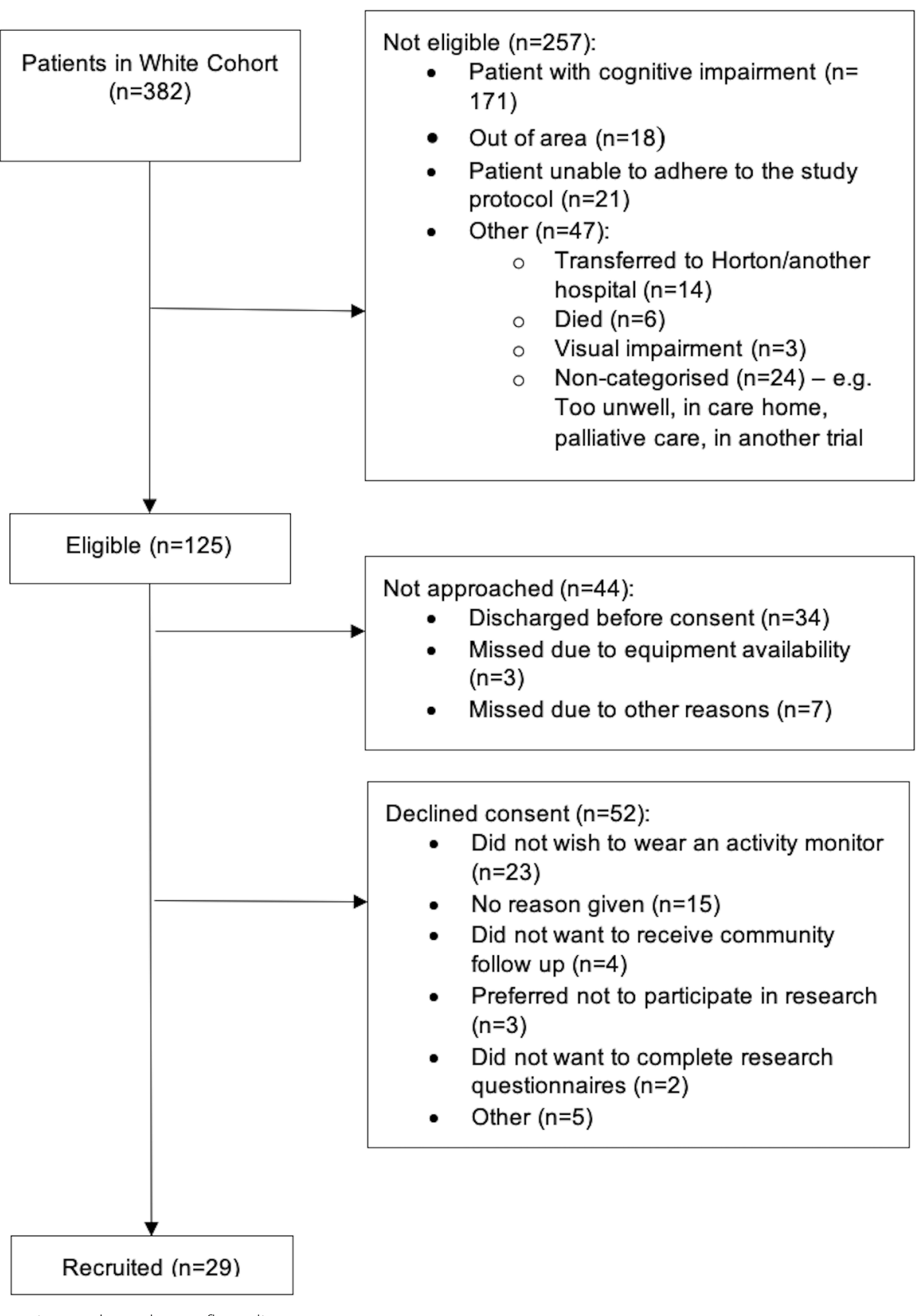

Fig. 3 Participant screening and enrolment flow diagram

Overall, participant wear of the Axivity monitor in the pendant system as defined for this study did not meet pre-defined thresholds for feasibility and acceptability.

\section{Activity data analysis}

The participants started wearing monitors after a median of 2 (IQR $1.8,5)$ days post-surgery and were discharged from the hospital after a median of 6 (IQR 4, 10.5) days post-surgery. Only four participants had complete 16-week ENMO data, whilst 2 participants had half or more data missing and were excluded from the mixed-effects modelling. Thirteen patients (median age 77.4 [IQR 70.5, 83.7] years, 92.3\% female) were included in the modelling procedure; 9 reported independent mobility at 16 weeks post-surgery, and the remaining 4 reported reliance on mobility aids or personal assistance. Demogpaphics for all participants and those who were included in the modelling analysis are presented in Table 1.

Each participant's ENMO ${ }_{W}$ recovery trend is shown in Fig. 4 (in blue and red). It can be observed that very little overlap exists between the groups from week 4, with 
Table 1 Participant characteristics

\begin{tabular}{|c|c|c|c|c|}
\hline & & & $\begin{array}{l}\text { Recruited Cohort } \\
(n=29 \text { unless otherwise stated) }\end{array}$ & $\begin{array}{l}n=13 \\
\text { (activity monitor group) }\end{array}$ \\
\hline \multicolumn{3}{|l|}{ Age, median [IQR] } & $76[71,82]$ & $77[71,84]$ \\
\hline \multicolumn{3}{|l|}{ Women, $n(\%)$} & $18(64.3)$ & $12(92.3)$ \\
\hline \multirow{2}{*}{$\begin{array}{l}\text { Pre-admission residence, } \\
\text { n (\%) }\end{array}$} & \multicolumn{2}{|l|}{ Own home } & $28(100)$ & $13(100)$ \\
\hline & \multicolumn{2}{|l|}{ Nursing home } & $0(0)$ & $0(0)$ \\
\hline \multirow[t]{4}{*}{ Pre-fracture mobility } & \multicolumn{2}{|l|}{ Freely mobile without aids } & $22(78.6)$ & $9(69.2)$ \\
\hline & \multicolumn{2}{|l|}{ Mobile outdoors with one aid } & $4(14.3)$ & $3(23.1)$ \\
\hline & \multicolumn{2}{|l|}{ Mobile outdoors with 2 aids or a frame } & $1(3.6)$ & $0(0)$ \\
\hline & \multicolumn{2}{|l|}{$\begin{array}{l}\text { Some indoor mobility, only mobilise outdoors } \\
\text { with help }\end{array}$} & $1(3.6)$ & $1(7.7)$ \\
\hline \multirow[t]{3}{*}{ Type of fracture, $n(\%)$} & \multicolumn{2}{|l|}{ Intracapsular } & 19 (67.9) & $9(69.27)$ \\
\hline & \multicolumn{2}{|l|}{ Extracapsular } & $8(28.6)$ & $4(30.7)$ \\
\hline & \multicolumn{2}{|l|}{ Sub-trochanteric } & $1(3.6)$ & $0(0)$ \\
\hline \multirow[t]{5}{*}{ Type of surgery, n (\%) } & \multicolumn{2}{|l|}{ Arthroplasty—total hip replacement } & $10(35.7)$ & $5(38.4)$ \\
\hline & \multicolumn{2}{|l|}{ Hemiarthroplasty } & $6(21.4)$ & $2(15.4)$ \\
\hline & \multirow[t]{3}{*}{ Fixation } & $\begin{array}{l}\text { Sliding hip } \\
\text { screw }\end{array}$ & $6(21.4)$ & $2(15.4)$ \\
\hline & & $\begin{array}{l}\text { Cannulated } \\
\text { screw }\end{array}$ & $2(7.1)$ & $2(15.4)$ \\
\hline & & IM nail (long) & $4(14.3)$ & $2(15.4)$ \\
\hline \multirow{4}{*}{$\begin{array}{l}\text { Type of anaesthesia, } n \\
(\%)\end{array}$} & \multicolumn{2}{|l|}{ General } & $20(71.4)$ & $11(84.6)$ \\
\hline & \multicolumn{2}{|l|}{ Intra-operative sedation } & $9(32.1)$ & $2(15.4)$ \\
\hline & \multicolumn{2}{|l|}{ Intra-operative nerve block } & $11(39.3)$ & $7(53.8)$ \\
\hline & \multicolumn{2}{|l|}{ Spinal } & $10(35.7)$ & $3(23.1)$ \\
\hline \multirow[t]{8}{*}{ Medical history, $n$ (\%) } & \multicolumn{2}{|l|}{ Myocardial infarction } & $2(7.1)$ & $2(15.4)$ \\
\hline & \multicolumn{2}{|l|}{ Heart failure } & $1(3.6)$ & $1(7.7)$ \\
\hline & Stroke/TIA & & $2(7.1)$ & $2(15.4)$ \\
\hline & Diabetes & & $0(0)$ & $0(0)$ \\
\hline & Renal dysfunction (moderate to severe) & & $0(0)$ & $0(0)$ \\
\hline & Respiratory disease & & $5(18.5)$ & $3(23.1)$ \\
\hline & $\begin{array}{l}\text { Rheumatoid arthritis or connective tissue } \\
\text { disease }\end{array}$ & & $3(10.7)$ & $2(15.4)$ \\
\hline & Malignancy & & $10(35.7)$ & $4(30.8)$ \\
\hline ASA Score, $n(\%)$ & । & & $3(10.7)$ & $1(7.7)$ \\
\hline & $\|$ & & $12(42.9)$ & $5(38.5)$ \\
\hline & III & & $6(21.4)$ & $2(15.4)$ \\
\hline & IV & & $5(17.9)$ & $3(23.1)$ \\
\hline & V & & $0(0)$ & $0(0)$ \\
\hline & Unknown & & $2(3.7)$ & $2(15.4)$ \\
\hline Other clinical factors & $\begin{array}{l}\text { Receipt community physiotherapy in 1st } \\
\text { month }\end{array}$ & & $8(28.6)^{*}$ & $3(23.1)$ \\
\hline & Pain/discomfort (baseline) ${ }^{*}$ & None & $13(48.1)$ & $6(46.2)$ \\
\hline & & Slight & $6(22.2)$ & $4(30.8)$ \\
\hline & & Moderate & $8(29.6)$ & $3(23.1)$ \\
\hline & & Severe & $0(0)$ & $0(0)$ \\
\hline & & Extreme & $0(0)$ & $0(0)$ \\
\hline
\end{tabular}


Table 1 Participant characteristics (Continued)

\begin{tabular}{|c|c|c|c|}
\hline & & $\begin{array}{l}\text { Recruited Cohort } \\
(n=29 \text { unless otherwise stated) }\end{array}$ & $\begin{array}{l}n=13 \\
\text { (activity monitor group) }\end{array}$ \\
\hline \multirow[t]{5}{*}{ Anxiety/depression (baseline)* } & None & $19(70.4)$ & $11(84.6)$ \\
\hline & Slight & $6(22.2)$ & $2(15.4)$ \\
\hline & Moderate & $1(3.7)$ & $0(0)$ \\
\hline & Severe & $0(0)$ & $0(0)$ \\
\hline & Extreme & $1(3.7)$ & $0(0)$ \\
\hline
\end{tabular}

ASA Score American Society of Anesthesiologists physical classification system score [23]

*Data available for 28 participants

those who self-reported mobilising independently at 16 weeks showing a much higher $\mathrm{ENMO}_{\mathrm{W}}$ from week 4 onwards (a participant reaching a maximum $\mathrm{ENMO}_{\mathrm{W}}$ of $13 g$ versus $6 g$ in each group). The average $\mathrm{ENMO}_{\mathrm{W}} \pm$ SE is presented in Fig. 5 in dashed lines, for each mobility outcome group. A significant increase in the average ENMO $_{\mathrm{W}}$, from $3.7 g(3.5,4.0 \mathrm{SE})$ to $8 g(7.4,9.4 \mathrm{SE})$, can be seen for the independently mobile group, versus an average $\mathrm{ENMO}_{\mathrm{W}}$ increase from $3.3 \mathrm{~g}(2.6,4.1 \mathrm{SE})$ to 5.0 $g$ (4.2, 5.7 SE, significantly lower), for the aid-dependent mobility group, from 1 to 16 post-operative weeks.

\section{Mixed-effects model}

Figure 5 shows the $E N M O_{W}$ (and 95\% Confidence Interval, CI) in solid lines, predicted from the baseline model, for each mobility outcome group. When considering data grouped at the participant level, the $E N M O_{W}$ is significantly different between the two groups (i.e. the $95 \%$
CI do not overlap) from week 5. The overlap at week 16 is caused by the lower amount of accelerometer data available in the latter weeks. This model indicates that accelerometer data can inform clinical staff about which patients are experiencing a faster or slower recovery in their mobility.

Finally, using the LRT statistic, it was verified that none of the other clinical factors (receipt of physiotherapy in the community post hospital discharge, pain/discomfort and anxiety/depression evaluated at the baseline questionnaire) provided a better model fit when added to the baseline model.

\section{Discussion}

Key results

Feasibility

We recruited 29 participants to this study, which assessed feasibility of using a pendant-worn activity monitor to better understand the recovery of mobility in

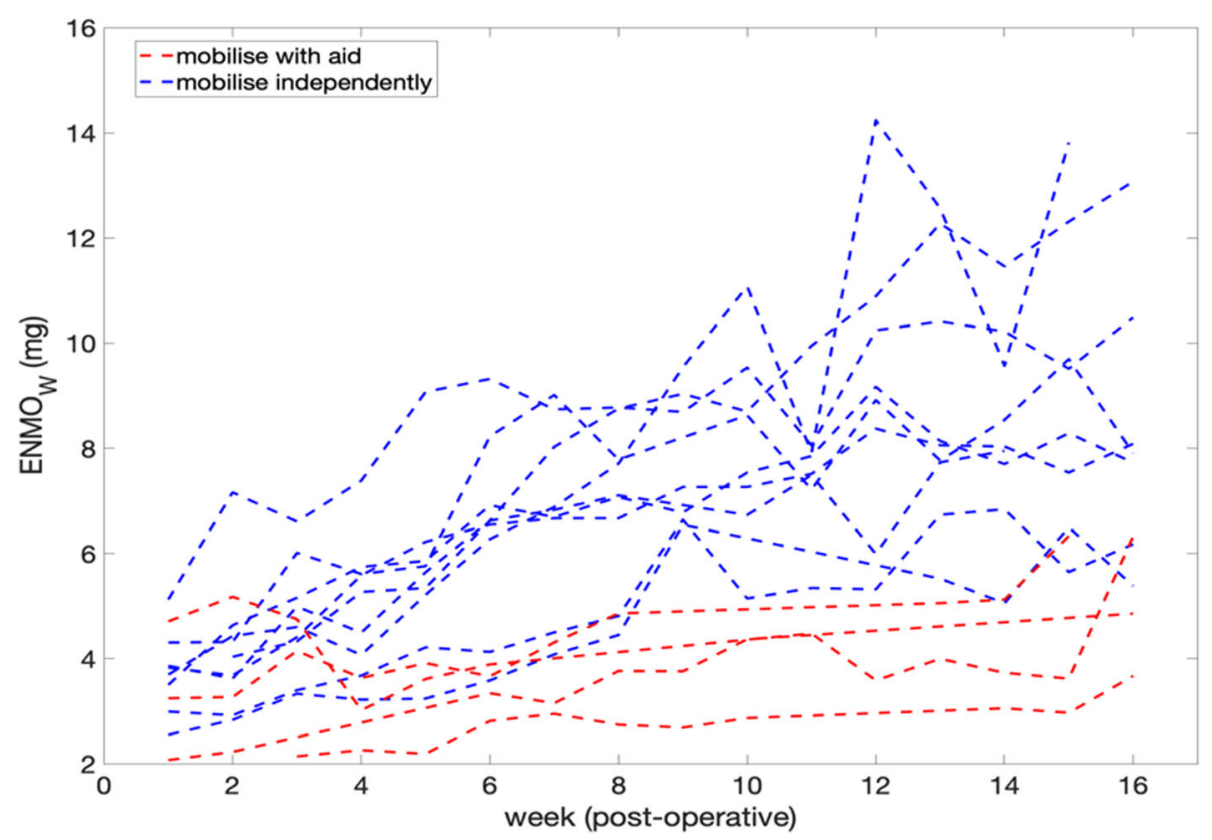

Fig. 4 Average ENMO per post-surgery week for each patient $\left(E N M O_{W}\right)$. Blue and red represent the participants groups with and without independent mobility at 16 weeks, respectively 


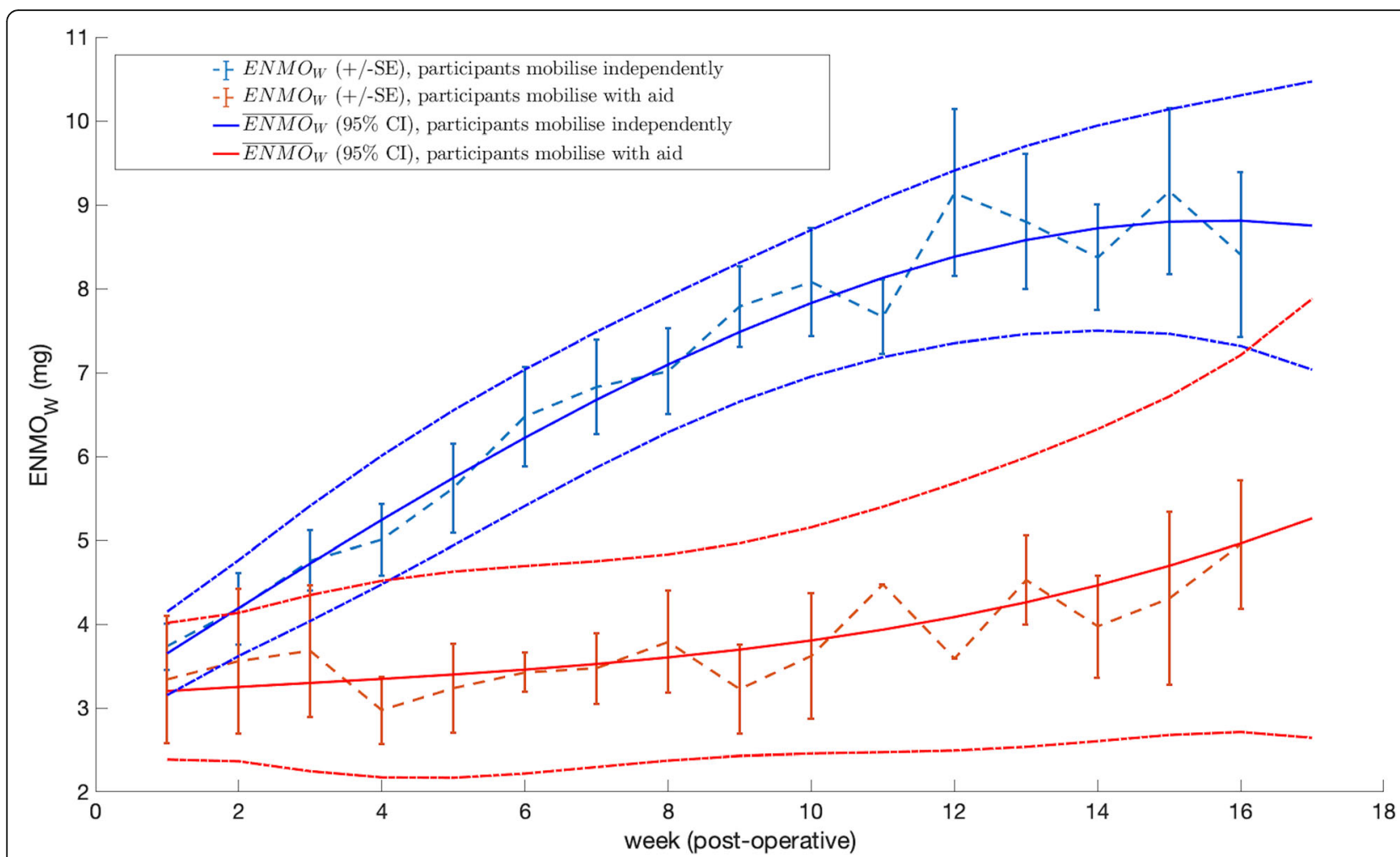

Fig. 5 Linear fixed effects model: polynomial fit $(n=3)$ from 6.30 am to $9.30 \mathrm{pm}$. The average $\mathrm{ENMO}_{\mathrm{W}} \pm$ SE per week are shown for each mobility outcome group in dashed lines. The ENMOW (and 95\% Cl) predictions of the mixed-effects model are also shown for each group in solid lines. Groups with and without independent mobility at 16 weeks are coloured blue and red, respectively

patients during the first 4 months of recovery post-hip fracture injury. We observed low consent rates and a high withdrawal rate. However, this study is novel in its collection of meaningful activity data through an axialworn activity monitor in this patient cohort, with previous studies being limited to monitors worn on the wrist or thigh $[2,3,7]$. By week 5, the predicted $\mathrm{ENMO}_{W}$ values were significantly different between the two mobility outcome groups (independently mobile versus not) meaning the model was able to inform the mobility outcome at 16 weeks. This identifies a potential opportunity for increased rehabilitative input for those demonstrating less activity at weeks $4-5$ of recovery, with the aim of improved mobility outcomes at 16 weeks.

Whilst we established feasibility of the monitor's ability to capture meaningful activity data when worn in this way, feasibility of using such a monitor for future research or clinical practice is limited by the limited feasibility of recruitment and retention of participants, as well as acceptability of the monitor observed in this study. The high withdrawal rate $(48 \%)$, coupled with some reports of a negative awareness of the monitor from those who completed the study protocol, demonstrates that the method of wearing the monitor employed in this study is not optimally suited to this demographic of the population. This adds an important contribution to the currently limited literature regarding optimal sensor placement and method of wear [24].

\section{Limitations}

This observational study excluded the large proportion of the hip fracture population who have concomitant cognitive impairment. The decision to exclude this group of patients was made owing to the potential safety implications that might arise from prolonged wear of a pendant system to house the activity monitor. The exclusion of this patient group limits the generalisability of our findings. However, whilst it is known that dementia is known to be a risk factor for poorer outcomes after hip fracture [25], there is some evidence to suggest that cognitive impairment does not have a significant impact on functional regain if rehabilitation is received [26, 27]. It should be noted that the median age of recruited participants $(76$, IQR 71, 82) was also markedly lower than the median age of the European hip fracture population (82, IQR 75, 87) [28].

Further limitations include the number of participants that we were able to recruit (29 participants) and the 
number of those with sufficient accelerometer data available to generate an activity trend model (13 participants). Participant self-report of mobility was used to define which participants were independently mobile and which were reliant on aids or assistance. Self-report is vulnerable to bias, and the study may have been improved by the inclusion of a mobility assessment, performed by a healthcare professional. However, an observational study of over 88,000 non-institutionalised older adults observed a concordance rate of $80 \%$ between self-report and professional assessment of mobility [29]. Results of statistical tests evaluating the significance of the addition of clinical factors to the baseline model should be interpreted cautiously, as the $\mathrm{ENMO}_{\mathrm{W}}$ dataset may be too small to derive conclusions from the LRT. The availability of data may have been further impaired by the design of the monitor.

In summary, although we were able to demonstrate a significant difference in the average activity between the group that gained full mobility at 16 weeks and the group that did not, this model is not generalisable to the full cohort of patients undergoing hip-fracture surgery, and could potentially be an overfitting to these data. Future work should evaluate its predictive performance on a held-out dataset.

\section{Conclusions}

This is the first study to our knowledge to investigate acceptability and feasibility of a pendant-worn activity monitor in this patient cohort. We have demonstrated that the ENMO metric may serve as a proxy for the level of physical activity of hip fracture patients during their post-operative recovery period. However, feasibility of recruitment, retention of participants and acceptability of the method of wearing the monitor in this study were notably limited. We have therefore not been able to demonstrate that this specific approach to monitoring activity of hip fracture patients would be feasible for larger-scale research studies. Further research into the use of activity monitors in this patient population should use minimally intrusive devices which are acceptable and safe for a more inclusive population, such as those with cognitive impairment.

\section{Supplementary information}

Supplementary information accompanies this paper at https://doi.org/10. 1186/s40814-020-00612-2.

\section{Additional file 1. Appendices.}

\section{Acknowledgements}

This work uses data provided by patients and collected by the NHS as part of their care and support and would not have been possible without access to this data. The NIHR recognises and values the role of patient data, securely accessed and stored, both in underpinning and leading to improvements in research and care. The authors thank Stephanie Wallis and the Trauma and Primary Care Research Nursing Teams of Oxford University Hospitals NHS Foundation Trust for their help with data collection and management. The authors thank Stephen Gerry and Dr Marco Pimentel for their suggestions in the statistical analysis plan for the activity data.

\section{Authors' contributions}

AF conceived the initial study idea and wrote the study protocol in collaboration with CV, PW, LT and MC. LA designed data collection tools, performed data analysis and interpretation of clinical data and drafted the first version of this manuscript. YC and MS performed data analysis and interpretation of the activity monitoring data. BL performed data management. BL, LA, and CA coordinated the study. All authors contributed to revisions of the manuscript and approved the final manuscript for submission.

\section{Funding}

This study was funded by the NIHR Oxford Biomedical Research Centre. The views expressed are those of the authors and not necessarily those of the NIHR or the Department of Health and Social Care. AF and MC are NIHR Senior Investigators.

\section{Availability of data and materials}

The datasets generated and analysed during the current study are not publicly available as further analyses and modelling of the data are currently being developed for publication, but datasets are available from the corresponding author on reasonable request.

\section{Ethics approval and consent to participate}

Research Ethics Committee approval was granted under the approval for the World Hip Trauma Evaluation Cohort Study (REC reference 11/LO/0927). All patients were required to give written consent to both the WHiTE and MoHIP studies in order to participate.

Consent for publication

Not applicable

\section{Competing interests}

None to declare

\section{Author details}

${ }^{1}$ Nuffield Department of Primary Care Health Sciences, Radcliffe Primary Care Building, Radcliffe Observatory Quarter, University of Oxford, Woodstock Road, Oxford OX2 6GG, UK. ${ }^{2}$ Institute of Biomedical Engineering, Department of Engineering Science, University of Oxford, Oxford, UK. ${ }^{3}$ Nuffield Department of Clinical Neurosciences, University of Oxford, Oxford, UK. ${ }^{4}$ Nuffield Department of Orthopaedics, Rheumatology and Musculoskeletal Sciences, University of Oxford, Oxford, UK.

Received: 30 January 2020 Accepted: 27 April 2020

Published online: 22 May 2020

\section{References}

1. Fernandez MA, Arnel L, Gould J, Mcgibbon A, Grant R, Bell P, et al. Research priorities in fragility fractures of the lower limb and pelvis: a UK priority setting partnership with the James Lind Alliance. BMJ Open. 2018;8:23301.

2. Taraldsen K, Sletvold O, Thingstad P, Saltvedt I, Granat MH, Lydersen S, et al. Physical behavior and function early after hip fracture surgery in patients receiving comprehensive geriatric care or orthopedic care - a randomized controlled trial. J Gerontol Ser A Biol Sci Med Sci. 2014;69A(3):338-45.

3. Schmal H, Holsgaard-Larsen A, Izadpanah K, Brønd JC, Madsen CF, Lauritsen J. Validation of activity tracking procedures in elderly patients after operative treatment of proximal femur fractures. Rehabil Res Pract. 2018 Jun 19;2018:1-9.

4. National Hip Fracture Database (NHFD) Annual report September 2018. 2018.

5. Boulton C, Bunning T, Hannaford J, Johansen A, Liddicoat M, Wakeman R. Recovering after a hip fracture: helping people understand physiotherapy in the NHS; 2018.

6. Althubaiti A. Information bias in health research: definition, pitfalls, and adjustment methods. J Multidiscip Healthc. 2016;9:211-7. 
7. Rowlands AV, Mirkes EM, Yates T, Clemes S, Davies M, Khunti K, et al. Accelerometer-assessed physical activity in epidemiology: are monitors equivalent? Med Sci Sports Exerc. 2018;50(2):257-65.

8. Nyman SR, Victor CR. Use of personal call alarms among communitydwelling older people. Ageing Soc. 2014 Jan 15;34(1):67-89.

9. Costa ML, Griffin XL, Achten J, Metcalfe D, Judge A, Pinedo-Villanueva R, et al. World Hip Trauma Evaluation (WHiTE): framework for embedded comprehensive cohort studies. BMJ Open. 2016;6(10):e011679.

10. Haywood KL, Griffin XL, Achten J, Costa ML. Developing a core outcome set for hip fracture trials. Bone Joint J. 2014 Aug 1;96-B(8):1016-23.

11. EuroQol EQ-5D-5 L [Internet]. [cited 2019 Aug 22]. Available from: https:// euroqol.org/eq-5d-instruments/eq-5d-5l-about/.

12. van Hees VT, Fang Z, Langford J, Assah F, Mohammad A, da Silva ICM, et al. Autocalibration of accelerometer data for free-living physical activity assessment using local gravity and temperature: an evaluation on four continents. J Appl Physiol. 2014.

13. Moore CG, Carter RE, Nietert PJ, Stewart PW. Recommendations for planning pilot studies in clinical and translational research. Clin Transl Sci. 2011 Oct; 4 (5):332-7.

14. Harris PA, Taylor R, Minor BL, Elliott V, Fernandez M, O'Neal L, et al. The REDCap consortium: building an international community of software platform partners. J Biomed Inform. 2019;95:103208.

15. Harris PA, Taylor R, Thielke R, Payne J, Gonzalez N, Conde JG. Research electronic data capture (REDCap) - a metadata-driven methodology and workflow process for providing translational research informatics support. J Biomed Inform. 2009 Apr 1;42(2):377-81.

16. Menai M, Van Hees VT, Elbaz A, Kivimaki M, Singh-Manoux A, Sabia S. Accelerometer assessed moderate-To-vigorous physical activity and successful ageing: results from the Whitehall II study. Sci Rep. 2017.

17. Bakrania K, Yates T, Rowlands A V., Esliger DW, Bunnewell S, Sanders J, et al. Intensity thresholds on raw acceleration data: Euclidean norm minus one (ENMO) and mean amplitude deviation (MAD) approaches. PLoS One. 2016;11(10).

18. Hildebrand M, Van Hees VT, Hansen BH, Ekelund U. Age group comparability of raw accelerometer output from wrist-and hip-worn monitors. Med Sci Sports Exerc. 2014;

19. Orive M, Anton-Ladislao A, García-Gutiérrez S, Las Hayas C, González N, Zabala J, et al. Prospective study of predictive factors of changes in pain and hip function after hip fracture among the elderly. Osteoporos Int. 2016 Feb 5;27(2):527-36.

20. Magaziner J, Simonsick EM, Kashner TM, Hebel JR, Kenzora JE. Predictors of functional recovery one year following hospital discharge for hip fracture: a prospective study. J Gerontol. 1990;45(3):M101-7.

21. Siu AL, Penrod JD, Boockvar KS, Koval K, Strauss E, Morrison RS. Early ambulation after hip fracture: effects on function and mortality. Arch Intern Med. 2006 Apr 10;166(7):766-71.

22. Salpakoski A, Törmäkangas T, Edgren J, Sihvonen S, Pekkonen M, Heinonen A, et al. Walking recovery after a hip fracture: a prospective follow-up study among community-dwelling over 60-year old men and women. Biomed Res Int. 2014:2014:289549.

23. Dripps $\mathrm{R}$, Lamont A, Eckenhoff J. The role of anesthesia in surgical mortality. JAMA. 1961:178(3):261-6.

24. Gupta A, Al-Anbuky A, McNair P. Activity classification feasibility using wearables: considerations for hip fracture. J Sens Actuator Networks. 2018;7(4):54.

25. Pioli G, Lauretani F, Pellicciotti F, Pignedoli P, Bendini C, Davoli ML, et al. Modifiable and non-modifiable risk factors affecting walking recovery after hip fracture. Osteoporos Int. 2016 Jun 20;27(6):2009-16.

26. Muir SW, Yohannes AM. The impact of cognitive impairment on rehabilitation outcomes in elderly patients admitted with a femoral neck fracture: a systematic review. J Geriatr Phys Ther. 2009;32(1):24-32.

27. Al-Ani AN, Flodin L, Söderqvist A, Ackermann P, Samnegård E, Dalén N, et al. Does rehabilitation matter in patients with femoral neck fracture and cognitive impairment? A prospective study of 246 patients. Arch Phys Med Rehabil. 2010 Jan;91(1):51-7.

28. Baker PN, Salar O, Ollivere BJ, Forward DP, Weerasuriya N, Moppett IK, et al. Evolution of the hip fracture population: time to consider the future? A retrospective observational analysis. BMJ Open. 2014;4(4):e004405.

29. Spitzer S, Weber D. Reporting biases in self-assessed physical and cognitive health status of older Europeans. Origo FM, editor. PLoS One. 2019;14(10):e0223526.

\section{Publisher's Note}

Springer Nature remains neutral with regard to jurisdictional claims in published maps and institutional affiliations.

\section{Ready to submit your research? Choose BMC and benefit from:}

- fast, convenient online submission

- thorough peer review by experienced researchers in your field

- rapid publication on acceptance

- support for research data, including large and complex data types

- gold Open Access which fosters wider collaboration and increased citations

- maximum visibility for your research: over $100 \mathrm{M}$ website views per year

At BMC, research is always in progress.

Learn more biomedcentral.com/submissions 